\title{
NOTAS SOBRE LA EVOLUCION DE LA BASE ECONOMICA EN LA REGION VALENCIANA
}

\author{
338.983:311 (46 Valencia)
}

por

\section{Ignacio Ballester Ros}

SUMARIO: I. INTRODUCCION.-II. LAS FUENTES Y LA TECNICA UTILIZADAS.-III. LA EVOLUCION DE LA ACTIVIDAD .ECONOMICA DE LA REGION: 1. LA PRODUCCIÓN AGRARIA Y PESQUERA. 2. LA PRODUCCIÓN INDUSTRIAL. 3. LA PRESTACIÓN DE SERVICIOS. 4. RESUMEN.

\section{INTRODUCCION}

En los últimos años se han realizado en España varios estudios de carácter estructural que suministran información básica sobre la interdependencia económica entre las distintas Regiones de un país. Su finalidad es, en cierto modo, similar a la que satisfacen las tablas «input-output» o las contabilidades regionales, aunque se trata de modelos más simplificados, que pueden elaborarse con información menos completa de las que necesitan las investigaciones apuntadas y cuyo estudio requiere también menor tiempo.

Estos trabajos se fundamentan en el estudio de la «base económica» de una Región, concepto que comprende el conjunto de actividades dedicadas a la obtención de bienes y prestación de servicios que habrán de ser vendidos, directa o indirectamente, fuera de la Región. En consecuencia, se califican como actividades económicas "básicas» aquellas que suministran unidades de producción o consumo fuera de la Región, y «no básicas», las que abaste- 
cen los mercados locales. Como es lógico, el nervio económico de una Región depende, en esta concepción, de las actividades básicas.

En otras palabras, la idea sustancial de la «base económica» es que el nivel de la actividad global de una Región viene determinado por el nivel de actividad de aquellos sectores de la actividad económica que se sostienen esencialmente por la demanda externa a la Región.

Estos trabajos permiten disponer de una información estructural ordenada con arreglo a un esquema original, conocer las verdaderas fuentes de renta y empleo de una Región y determinar cuáles son las actividades más productivas, cuyo desarrollo debe impulsarse en beneficio de la economía de la Región.

Tal vez, el primero de los estudios de esta naturaleza que se elaboró en España fue «La estructura de la base económica de Cataluña", obra del profesor Ramón Trías FarGas, que se publicó en 1965.

Siguiendo esta pauta, y bajo la dirección del propio profesor Trfas Fargas, José T. RAGa GiL realiza un primer trabajo que presenta, en 1966, como tesis doctoral en la Universidad de Valencia, en el que analiza, desde una perspectiva estática y a corto plazo, las actividades económicas que configuran la base económica de la Región Valenciana.

Continúa sus estudios sobre esta materia y en 1976 publica su nueva obra, titulada "Crecimiento de la base económica en el País Valenciano. Análisis estructural dinámico", en el que registra los cambios estructurales y mide la tasa de crecimiento de la economía valenciana en sus distintos sectores.

En esta obra, el doctor RAGA GIL formula un planteamiento general del análisis regional y explica el concepto y las técnicas a aplicar para la determinación de la base económica, expone los perfiles económicos y su evolución en la Región Valenciana y entra de lleno en la elaboración de la base económica de la Región y su evolución, esencialmente en el período 1960-1971, que precisamente corresponde a la ejecución de los dos primeros Planes de Desarrollo en España, tras el Plan de Estabilización.

El reconocimiento de los Entes preautonómicos y el régimen de autonomía para las Regiones, reconocido en la nueva Constitución española, que se va a someter a referéndum nacional, y el hecho de que dispongamos de este notable trabajo del doctor RAGA 
GIL, que nos permite conocer la evolución reciente y las perspectivas que se abren a la economía valenciana, cara a su futuro, justifican que le dediquemos esta crónica, resaltando las características y las conclusiones más importantes que se deducen de este estudio.

\section{LAS FUENTES Y LA TECNICA UTILIZADAS}

Las fuentes utilizadas por el doctor RAGA GIL para la elaboración de este estudio han sido, de una parte, las publicaciones del Servicio de Estudios del Banco de Bilbao sobre «La renta nacional de España y su distribución provincial», correspondiente a los años 1955, 1957, 1960 y 1971, y, de otra, el «Censo industrial de España», de 1958, y la "Estadística industrial de España», de 1971, publicaciones ambas del Instituto Nacional de Estadística.

En la obra que comentamos, el autor ha expuesto ampliamente los procedimientos aplicados en distintos países para determinar la base económica regional y la dinámica de su evolución.

El procedimiento utilizado por el autor consiste en determinar con referencia a cada sector, subsector o actividad económica, el coeficiente de locación en los años citados, tomando como base el método del valor añadido y el del empleo.

Para determinar el citado coeficiente mediante el valor añadido aplica la siguiente fórmula:

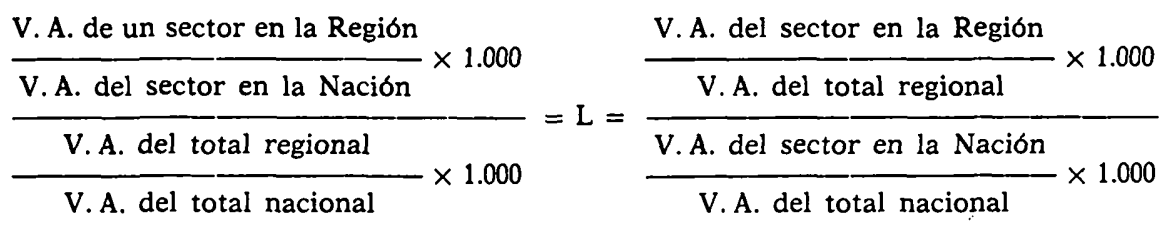

El valor $\mathrm{L}$ indica la posición de cada sector, subsector o actividad en el sistema económico regional. Se basa en la relación que existe entre la producción del sector, subsector o actividad en la Región y en la Nación, bajo un denominador común que es la importancia de la Región en el conjunto nacional. La primera fracción expresa la relación entre el sector considerado en la Región y el total de la economía regional. La segunda expresa lo que supone ese mismo sector tomado en el ámbito nacional en compa- 
ración con la economía nacional en su conjunto. El resultado de ambas fracciones es idéntico.

Para el método del empleo se utiliza la misma fórmula, sustituyendo el valor añadido por el empleo.

Estos coeficientes de locación, así obtenidos, tienen un carácter estático, referidos a cada uno de los años considerados.

El autor utiliza como complemento de éste otro coeficiente dinámico "D», referido al período 1971-1960, que se obtiene para cada sector, como resultado de una fracción cuyo numerador es el coeficiente de locación estático del sector en el año más próximo -en este estudio, 1971- y el denominador el coeficiente de locación del mismo sector en el año más remoto, 1960.

En el Apéndice Estadístico hemos consignado, con ligeras modificaciones de ordenación, los coeficientes de locación obtenidos por el método del valor añadido de los sectores y del desglose, por subsectores, de la producción industrial y minera y de la producción agraria en los cuatro años considerados; y los coeficientes dinámicos «D" para los mismos sectores y el desglose de la producción industrial y minera en el período 1960-1971, tomándolos de la obra comentada, con respecto a la Región, a cada una de las Provincias que la forman y al resto de España.

\section{LA EVOLUCION DE LA ACTIVIDAD ECONOMICA DE LA REGION}

La Región Valenciana tiene una extensión superficial de 23.295 kilómetros cuadrados y una población de 3.411.218 habitantes, según el Padrón municipal de 1975, lo que representa el 4,6 por 100 de la superficie nacional y casi el 10 por 100 de la población española.

Del análisis de los coeficientes de locación, estáticos y dinámicos, consignados en el Apéndice Estadístico, puede deducirse la evolución seguida por la actividad económica regional en el período 1955-1971 y su tendencia dinámica en 1960-1971, por sectores y sub. sectores, y permite destacar aquellos que merecen calificarse de básicos, en el sentido expuesto en la Introducción, en los que se apoya la economía de la Región. 
En ambos coeficientes, los valores iguales o superiores a la unidad son los que permiten calificar de básico o exportador al sector o subsector a que se refieren en cada uno de los cuatro momentos que se consideran en el coeficiente de locación estático, así como en el dinámico.

Analicemos, por separado, los tres sectores económicos.

\section{LA PRODUCCIÓN AGRARIA Y PESQUERA}

El doctor RAGA GIL traza el perfil económico del sector agrario distinguiendo tres zonas de cultivos. La primera, de cultivo hortícola intensivo, se extiende en la llanura litoral, al Norte y Sur de la ciudad de Valencia, sin adentrarse hacia el interior más de siete kilómetros, y constituye la Huerta de Valencia. En ella se dan los más variados cultivos hortícolas, entre ellos los de exportación. Predomina la mano de obra directa, pues el minifundio generalizado impide la utilización de maquinaria. El sistema de regadío, la aplicación de nuevas técnicas de cultivo, el empleo de fertilizantes, la selección de semillas y la plena dedicación del agricultor valenciano determinan altos rendimientos en sus cosechas.

La segunda zona, la de los frutales - primordialmente la naranja y otros cítricos- se extiende por las Comarcas de Vinaroz y Benicarlo, la Plana de Castellón, Sagunto y parte del valle del Palancia, los Campos de Liria y Chiva, las Riberas Alta y Baja, la Comarca de Játiva, la Huerta de Gandía, La Marina y Alicante y su contorno, penetrando hacia el interior de treinta a cuarenta kilómetros. Constituye en muchos términos municipales un monocultivo, que no exige tanta dedicación como en la Huerta. Su elevada rentabilidad de antaño ha decrecido. En parte, el productor naranjero no es labrador, sino el rentista o el industrial que invierte en la tierra para obtener un beneficio, pero vive en la ciudad.

Tiende a desaparecer el agricultor de la primera zona para asimilarse al de la segunda; así, en la Huerta de Valencia se ha introducido el cultivo del naranjo, que se riega con agua de las acequias del Turia, restando superficie al cultivo hortícola propiamente dicho, atendido por descendientes de la anterior generación de agricultores, que hoy tienen como principal actividad la industria. 
La tercera zona es la tradicional de secano, que se extiende por el resto de la Región, y comprende los terrenos menos fértiles y de mayor dificultad para su transformación en regadío, con cultivos de frutos secos y viñedos.

Se han operado profundas transformaciones en la agricultura valenciana. Ha perdido la hegemonía en cultivos tradicionales, como el del arroz, y otros que han perdido valor en el mercado. La elevación de salarios y de los costes de producción y las restricciones de crédito dificultan su desarrollo. El agricultor que antes se preocupaba sólo de producir más y mejor y de combinar las posibilidades productivas para conseguir una mayor productividad de la tierra, hoy tiene que preocuparse más de vender que de producir. La comercialización es fundamental para asegurar mercados que absorban la producción agrícola de exportación, con precios remuneradores.

La evolución en el período considerado se produce con marcada irregularidad, si bien, en conjunto, la producción agrícola, forestal y pecuaria se mantiene como básica en el período considerado.

El desglose de la actividad agraria, que figura en el Apéndice, permite conocer mejor esta evolución. La Región Valenciana y cada una de sus tres Provincias son fundamentalmente exportadoras tan sólo en el grupo de "hortalizas, frutas y patatas"; en los restantes grupos la situación es deficitaria, en especial por lo que se refiere a cereales, leguminosas, leche y productos forestales.

En 1960-1971 ha perdido fuerza este sector en la Región, mientras que en el resto de España su tendencia es positiva, tal vez por el desarrollo de los planes de colonización y de extensión de los regadíos fuera de la Región.

La actividad pesquera tiene un carácter deficitario en la Región y en la Provincia de Valencia. En Alicante se muestra como básica en 1957 y 1971, y en Castellón en 1955, 1960 y 1971, con un elevado coeficiente en este último año, lo que justifica que atienda el consumo interior de la Región y el abastecimiento, en parte, del mercado nacional, lo que no quiere decir que la Región sea autosuficiente. La tendencia en 1960-1971, reflejada en el coeficiente dinámico, es creciente y básica en toda la Región. 


\section{LA PRODUCCION INDUSTRIAL}

En la Región Valenciana coexisten dos tipos de empresas industriales, de dimensión dispar. De una parte, grandes empresas, líderes de subsectores en el ámbito nacional, con una producción competitiva, orientada a los mercados exteriores, con buenas fuentes de financiación, a las que han llegado recientemente inversiones extranjeras.

El segundo tipo, muy extendido en la Región, lo constituye el taller y la pequeña industria, creada muchas veces por el empresario individual, sin facilidades de crédito, que aspira sólo a abastecer mercados locales. Nacidas al calor de una coyuntura económica favorable, muchos de estos empresarios no aplican sus beneficios a la modernización del equipo y al desarrollo de su industria, sino a la adquisición de parcelas agrícolas y de terrenos susceptibles de revalorización. La demanda de trabajadores por la agricultura, con elevados salarios, contribuye a crear una insuficiencia de empleo en la industria o lo frena en los ciclos agrícolas que exigen más mano de obra. Este fraccionamiento empresarial supone un freno a la actividad industrial, con las dificultades planteadas a la pequeña y mediana empresa en orden a lograr una producción exportadora.

Los principales núcleos industriales de la Región son, en la Provincia de Castellón, Vinaroz y Benicarló, y Castellón y su entorno, con Burriana y Villarreal, Lucena del Cid, Alcora, Onda y Vall de Uxó. En la Provincia de Valencia, Sagunto, Valencia y su zona de influencia, Liria y Puebla de Vallbona, Chiva, Requena y Utiel, Almusafes y Benifayó, Sueca y Carlet, Alcira y Gandía, Játiva y Onteniente. Y en la Provincia de Alicante, Denia y. Benisa, Alcoy y Jijona, Elda y Novelda, Alicante y su entorno, Elche, Orihuela y Torrevieja.

En el conjunto de la Región y en la Provincia de Valencia, la producción industrial y minera tiene un carácter básico en los momentos extremos del período estudiado. En Alicante ofrece tal carácter de 1957 a 1971. En Castellón no lo alcanza, si bien registra un coeficiente de locación más alto en 1971. La tendencia es, sin embargo, positiva para toda la Región en 1971, lo que contrasta con el resto de España, que ofrece en dicho año un coeficiente dinámico inferior a la unidad. 
Parece muy conveniente analizar el desglose por subsectores de este sector económico. La minería es deficitaria en la Región, aunque tiende a mejorar en 1971. Los coeficientes más altos corresponden a lo largo del período a Alicante y Castellón, aunque en 1971 destacan Alicante y Valencia. Las reservas minerales son pobres en carbones y minerales metálicos. Se registra una actividad apreciable en la extracción de piedra, arcilla y arena, más concretamente, con carácter básico, en los caolines, en toda la Región y especialmente en la Provincia de Valencia, y en la sal en la Provincia de Alicante, lo que contrasta con el carácter exportador de la minería en el resto de España. Sin embargo, el coeficiente dinámico es positivo en toda la Región en 1960-1971, en especial en la Provincia de Valencia.

La alimentación es un subsector básico en 1955 en la Región y en la Provincia de Valencia. En Alicante es básica en 1955-60, y en Castellón no alcanza tal carácter. Las actividades básicas son los molinos arroceros, las industrias de extractos y condimentos, piensós compuestos, confituras y productos del cacao, las cárnicas y las de productos de panadería, destacando las Provincias de Valencia y Alicante, como el resto de España. La elaboración de bebidas tiene carácter básico en la Provincia de Valencia, y la industria de tabacos en las de Alicante y Valencia, aunque con tendencia decreciente.

La industria textil es básica en la Región de 1957 a 1971 y en Alicante durante todo el período considerado. El coeficiente dinámico es positivo para la Región y las Provincias de Castellón y Valencia.

La industria de la piel, calzado y confección es una actividad básica muy importante en la Región y en las Provincias de Alicante y Castellón a lo largo del período, aunque con una tendencia decreciente. En la Provincia de Valencia lo es en 1957 y 1971. Por actividades, destacan la industria del calzado en las Provincias de Castellón y de Alicante - Vall de Uxó y Elda y otras poblaciones del valle del Vinalopó, respectivamente-, con empresas de un tamaño superior a las del resto de España; la confección de prendas de vestir en la Provincia de Castellón, y otras confecciones textiles en las de Alicante y Valencia. El coeficiente dinámico es positivo sólo en la Provincia de Valencia. Se registra una pérdida de las condiciones exportadoras de la Región, por el establecimien- 
to de industrias similares en los polos de desarrollo y por las restricciones a la importación por parte de los Estados Unidos.

La industria de madera y corcho es básica en la Región y en la Provincia de Valencia. También son básicas las Provincias de Castellón en 1960 y 1971 y la de Alicante de 1955 a 1960. La actividad más importante es la fabricación de muebles y sus accesorios. El tamaño empresarial es muy superior al promedio nacional. El coeficiente dinámico es positivo para la Región y las Provincias de Castellón y Valencia.

La industria del Papel, Prensa y Artes Gráficas se mantiene básica en la Región en 1955 y 1957; en la Provincia de Alicante, de 1955 a 1960, y en la de Valencia en 1955, 1957 y 1971. Por actividades, destacan como básicas las de fabricación y manipulados de papel y cartón y la de planimetría y litografía en la Región y en la Provincia de Valencia, si bien es deficitaria en Artes Gráficas. El coeficiente dinámico es positivo en la Región y las Provincias de Castellón y Valencia.

La industria química es básica en la Región y en la Provincia de Valencia en 1955; en la de Alicante en 1957, y en la de Castellón en 1971, debido al reciente establecimiento de un complejo petroquímico.

Las industrias metálicas básicas son deficitarias en la Región, salvo la Provincia de Valencia en 1971, como consecuencia del establecimiento de los Altos Hornos del Mediterráneo. Analizando las distintas actividades de este sector puede afirmarse que son básicas la industria del aluminio y otros metales no férreos en Alicante y la del cobre en Valencia. El sector en conjunto adquiere un carácter exportador en 1971.

En el subsector de fabricación y reparación de productos metálicos destacan como básicas las actividades de ferretería, herrería y fumistería en la Provincia de Alicante, y las de recubrimientos metálicos, otros productos metálicos y artículos domésticos en la de Valencia.

En el subsector de construcción y reparación de maquinaria no eléctrica cabe distinguir como básicas las industrias de fabricación de maquinaria agrícola, con la más importante empresa española y un coeficiente de locación 30 , de maquinaria para las industrias vinícola y oleícola y para la.piel y calzado, con elevados coeficientes en la Provincia de Alicante, así como la de maquinaria 
para la piel y el caucho en la de Castellón, y la de maquinaria para la industria conservera en las de Valencia y Castellón.

En cuanto al subsector de construcción y reparación de maquinaria eléctrica destacan como básicas las de aparatos de medida y maquinaria y material diverso en la Provincia de Alicante, y la de material de transmisión eléctrica en la de Valencia.

Respecto a la construcción y reparación de material de transporte, son de señalar como básicas las de construcción y reparación de equipo ferroviario y construcción naval en la Provincia de Valencia y la reparación de automóviles en las de Valencia y Castellón.

La industria de la construcción y sus materiales es deficitaria en la Región en el subsector de edificación y obras públicas, si bien registra una franca recuperación en 1960-1971, debido a la ejecución del Plan Sur, con las vías de acceso a la ciudad de Valencia y de la autopista del Mediterráneo.

La principal componente del subsector de minerales no metálicos es la actividad de la cerámica, vidrio y cemento, muy arraigadas en las Provincias de Castellón -Onda y Alcora- y Valencia -Manises-, si bien últimamente las tres Provincias son exportadoras, como igualmente lo son en cales hidráulicas.

La industria del agua, gas y electricidad es básica en la Región en 1957 y en la Provincia de Valencia en 1955 y 1957, destacando solamente la del gas.

Con referencia a otras industrias fabriles conviene señalar que la Región se halla a la cabeza del mercado nacional en la producción de juguetes y artículos de deportes en la Provincia de Alicante, con un coeficiente 15 , y en la de Valencia. Sin embargo, tiende a disminuir el coeficiente dinámico, debido a la crisis del juguete como consecuencia de la contracción del mercado exterior y de la competencia de Japón y China en el mercado internacional e incluso en el nacional.

\section{LA PRESTACION DE SERVICIOS}

El comercio es una actividad muy importante, tanto por lo que se refiere a los productos agrícolas como a los industriales. Sabido es que los productos agrícolas se pagan por el comerciante al contado, en efectivo, en el momento de la contratación, con el fruto 
pendiente en el árbol o en la planta. Los costes de almacenamiento, la construcción de centrales hortofrutícolas, la elevación de salarios para la preparación y envase de los productos, el endurecimiento del mercado monetario y las dificultades del mercado internacional, de manera especial las trabas impuestas por el Mercado Común, a cuyos países se dirige gran parte de esta corriente, dificultan esta actividad, que es básica para toda la Región en el período de referencia y cuyo coeficiente dinámico es positivo en 1960-1971.

La actividad de transportes es correlativa a la comercial y básica en toda la Región, en especial en las Provincias de Castellón y Valencia. El tradicional transporte de exportación de los productos agrícolas por ferrocarril o por mar ha sido sustituido en gran parte por el transporte por carretera, por contenedores, hasta los países receptores, sirviendo, al propio tiempo, a otras ramas que, como la de cerámica, se han expansionado en el período de referencia. También se utiliza el transporte aéreo, desde Manises, para el transporte de productos muy delicados y perecederos.

En orden al Ahorro, Banca y Seguros, que refleja la fuerza del mercado, conviene subrayar que la Región se ha transformado de una zona de ahorro en otra de inversión, creando un mercado de capitales al servicio de su actividad económica; de aquí el desarrollo bancario registrado en toda la Región en 1960-1971.

Tal vez pudiera destacarse una situación deficitaria de los servicios públicos en una Región en la que predomina la iniciativa privada, si bien se van cubriendo adecuadamente. Los servicios personales y profesionales son básicos, de manera especial en Alicante y Valencia.

La explosión demográfica de la Región, con fuertes movimientos de inmigración, correlativos al desarrollo de una actividad económica básica, han presionado fuertemente el mercado de viviendas, un alto porcentaje de las cuales han sido adquiridas en propiedad mediante la amortización a plazo diferido. Complemento de esta actividad ha sido la construcción de urbanizaciones turísticas a lo largo del litoral de las tres Provincias, de manera especial en Alicante y Castellón. El desarrollo del turismo receptivo e interior ha promovido, a su vez, la construcción y puesta en explotación de hoteles y establecimientos de hostelería en las grandes ciudades y en las urbanizaciones turísticas. 


\section{Resumen}

El futuro de la economía valenciana se basa en los tres sectores analizados. En el sector agrario se apoya en la producción hortícola - patata, cebolla, tomate, melón y otros- y de frutales -naranjo y otros cítricos, melocotones y otros-, frutos secos - almendro-, uvas y vinos.

En el sector industrial se apoya en la industria de productos alimenticios - preparación de carnes, jugos y zumos, arroz, condimentos, chocolates, galletas y pastas-, elaboración de bebidas y de tabacos, fabricación de muebles, confección de calzado y de prendas de vestir, fabricación y manipulados de papel y cartón, petroquímica, siderurgia, metalurgia del aluminio y del cobre, fabricación de maquinaria para la industria, de material eléctrico e instrumentos de precisión, productos metálicos y artículos domésticos, construcción y reparación de equipo ferroviario, construcción naval, edificación y construcción, cerámica, loza y vidrio, juguetes y artículos de deportes.

En el sector de servicios se apoya en una actividad comercial en gran escala, en unos transportes en plena evolución, transformación y desarrollo, en unos servicios bancarios cada día más efectivos, si bien se considera necesaria la apertura de una bolsa de valores, como mercado de capitales; en unos planes de construcción de viviendas que permita atender las necesidades de la población residente e inmigrada y al movimiento turístico, juntamente con las igualmente necesarias instalaciones turísticas que atiendan las corrientes exterior e interior, $y$ en unos servicios públicos, personales y profesionales, que completen con suficiencia la atención de las necesidades de la población en el marco de la actividad económica de la Región. 


\section{APENDICE ESTADISTICO}

\section{COEFICIENTES DE LOCACION SOBRE EL VALOR ANADIDO DE LOS SECTORES}

\begin{tabular}{|c|c|c|c|c|}
\hline & 1955 & 1957 & 1960 & 1971 \\
\hline \multicolumn{5}{|l|}{ Pais Valenciano } \\
\hline 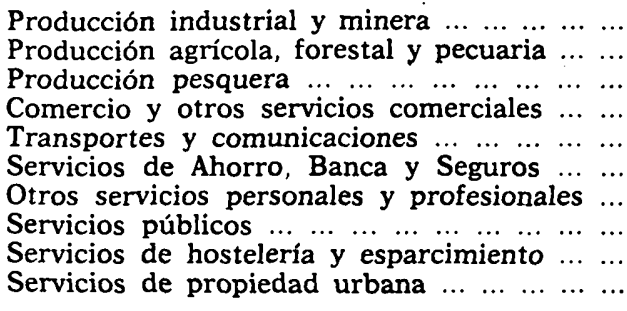 & $\begin{array}{l}1,030 \\
1,026 \\
0,473 \\
0,984 \\
1,109 \\
0,910 \\
1,038 \\
0,749 \\
0,881 \\
1,353\end{array}$ & $\begin{array}{l}0,934 \\
1,191 \\
0,477 \\
1,059 \\
1,053 \\
0,958 \\
1011 \\
0,750 \\
0,815 \\
1,280\end{array}$ & $\begin{array}{l}0,860 \\
1,398 \\
0,467 \\
1205 \\
0,920 \\
0,910 \\
0,889 \\
0,806 \\
0,775 \\
1.027\end{array}$ & $\begin{array}{l}1,039 \\
1,051 \\
0,844 \\
1,063 \\
0,937 \\
0,914 \\
0,969 \\
0,800 \\
0,848 \\
1,110\end{array}$ \\
\hline \multicolumn{5}{|l|}{ Provincia de Alicante } \\
\hline 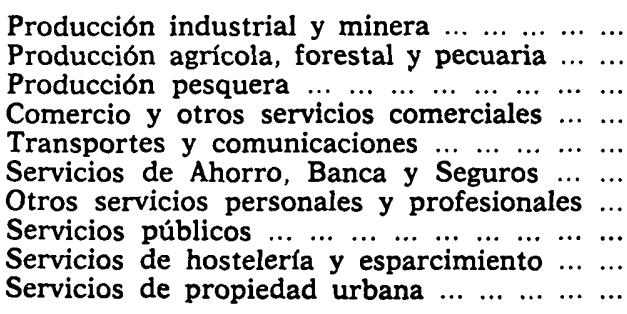 & $\begin{array}{l}0,124 \\
1,103 \\
0,929 \\
1096 \\
0,680 \\
1,206 \\
0,940 \\
0,529 \\
1,075 \\
0,930\end{array}$ & $\begin{array}{l}1,150 \\
0,937 \\
1018 \\
0,893 \\
1,212 \\
1,076 \\
1,045 \\
0621 \\
1,069 \\
0,987\end{array}$ & $\begin{array}{l}1,133 \\
0,986 \\
0,976 \\
0,891 \\
0,950 \\
0997 \\
0,993 \\
1,025 \\
1,061 \\
0,864\end{array}$ & $\begin{array}{l}1,144 \\
0,882 \\
1,465 \\
1,068 \\
0,790 \\
0,838 \\
0,831 \\
0,605 \\
1,244 \\
1,059\end{array}$ \\
\hline \multicolumn{5}{|l|}{ Provincia de Castellon } \\
\hline 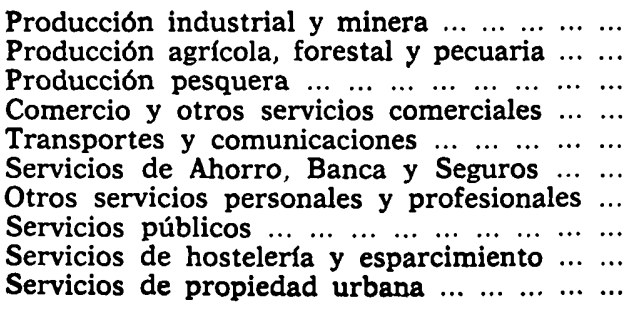 & $\begin{array}{l}0,776 \\
1,493 \\
1,688 \\
1,124 \\
0,748 \\
0,889 \\
0,010 \\
0,906 \\
0,924 \\
0,778\end{array}$ & $\begin{array}{l}0,793 \\
0,793 \\
0,793 \\
0,955 \\
0,818 \\
0,531 \\
0,883 \\
0,833 \\
0,479 \\
0,688\end{array}$ & $\begin{array}{l}0721 \\
2,057 \\
1,473 \\
0,767 \\
0,612 \\
0,615 \\
0554 \\
0,645 \\
0,506 \\
0,881\end{array}$ & $\begin{array}{l}0,922 \\
1,723 \\
2,306 \\
1,037 \\
0,890 \\
0,617 \\
0,582 \\
1,053 \\
0,671 \\
1,094\end{array}$ \\
\hline \multicolumn{5}{|l|}{ Provincia de Valencia } \\
\hline 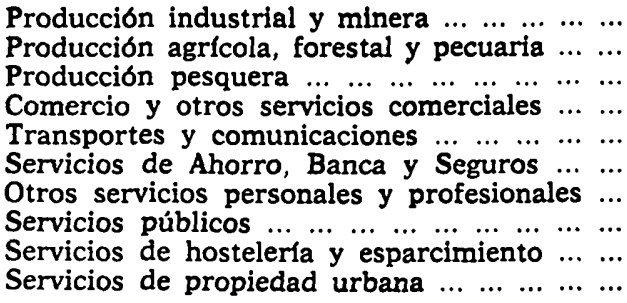 & $\begin{array}{l}1,066 \\
0,912 \\
0,088 \\
0,919 \\
1,325 \\
0,811 \\
1,087 \\
0,796 \\
0,807 \\
1,605\end{array}$ & $\begin{array}{l}0,899 \\
1,184 \\
0,088 \\
1,118 \\
1,051 \\
1004 \\
1,025 \\
0,771 \\
0,805 \\
1,478\end{array}$ & $\begin{array}{l}0,800 \\
1,404 \\
0102 \\
1,362 \\
0,971 \\
0,940 \\
0,845 \\
0,776 \\
0735 \\
1,109\end{array}$ & $\begin{array}{l}1,010 \\
0,987 \\
0,207 \\
1,065 \\
1,020 \\
1,015 \\
1,123 \\
0,842 \\
0,685 \\
1,137\end{array}$ \\
\hline
\end{tabular}


DESGLOSE DE LA PRODUCCION INDUSTRIAL Y MINERA

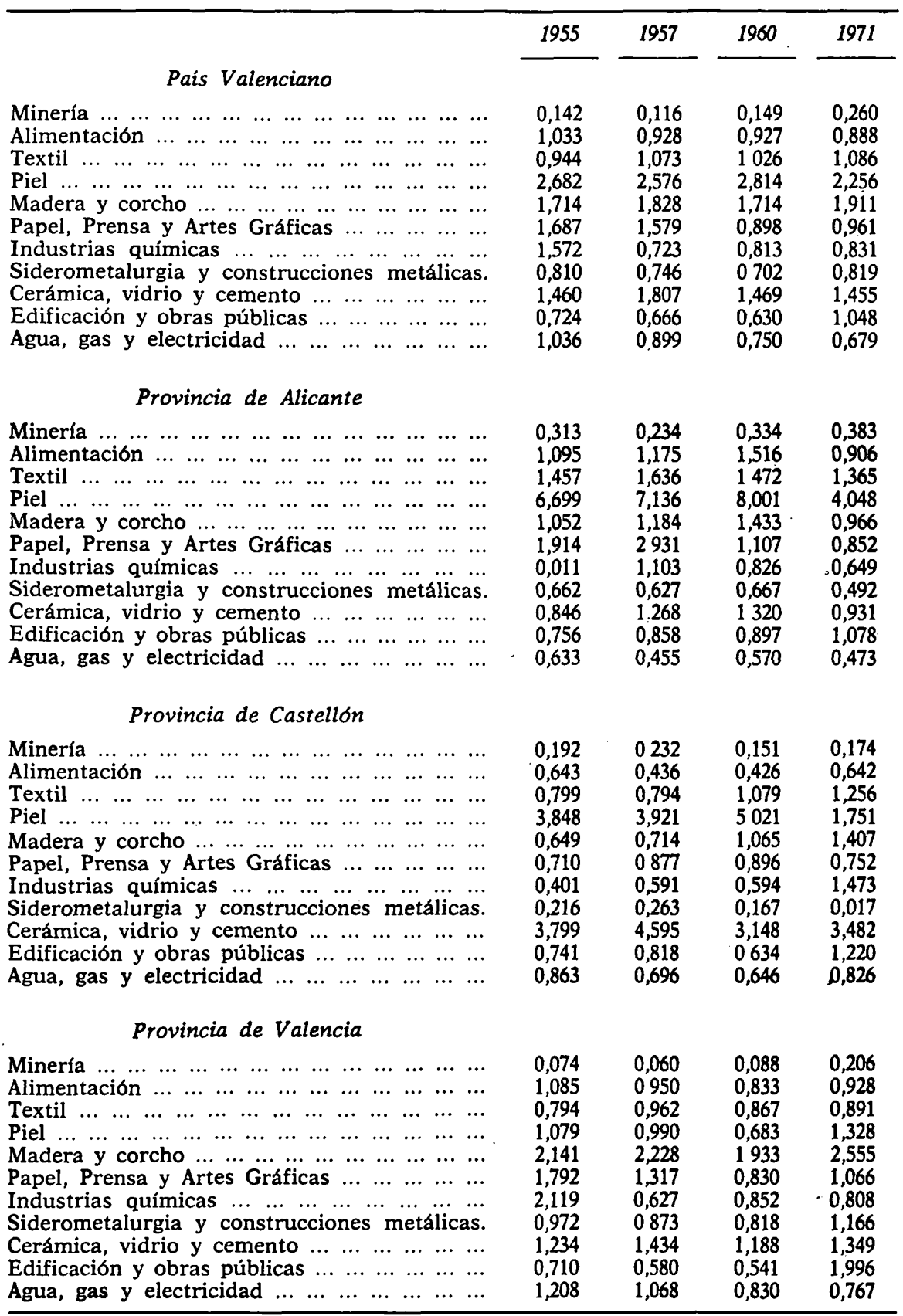




\section{RESTO DE ESPANA}

COEFICIENTES DE LOCACION SOBRE EL VALOR ANADIDO DE LOS SECTORES

\begin{tabular}{|c|c|c|c|c|}
\hline & 1955 & 1957 & 1960 & 1971 \\
\hline Producción industrial y minera $\ldots \ldots \ldots \ldots$ & 0,997 & 1,006 & 1,014 & 0,996 \\
\hline Producción agrícola, forestal y pecuaria ........ & 0,997 & 0,982 & 0,958 & 0,995 \\
\hline $\begin{array}{lllllllll}\text { Producción pesquera } & \ldots & \ldots & \ldots & \ldots & \ldots & \ldots & \ldots & \ldots\end{array}$ & 1,049 & 1050 & 1,055 & 1,015 \\
\hline Comercio y otros servicios comerciales ... ... & 1,001 & 0,994 & 0,978 & 0,994 \\
\hline $\begin{array}{llllll}\text { Transportes y comunicaciones } & \ldots & \ldots & \ldots & \ldots & \ldots\end{array}$ & 0,990 & 0,995 & 1,008 & 1,006 \\
\hline Servicios de Ahorro, Banca y Seguros ... ... & 1,008 & 1,004 & 1009 & 1,008 \\
\hline Otros servicios personales y profesionales ... & 0,996 & 0,999 & 1,017 & 1,003 \\
\hline 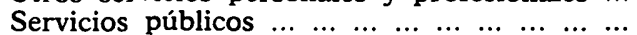 & 1,008 & 1,024 & 1,020 & 1,019 \\
\hline Servicios de hosteleria y esparcimiento ........ & 1,011 & 1018 & 1,023 & 1,015 \\
\hline $\begin{array}{llllll}\text { Servicios de propiedad urbana } & \ldots & \ldots & \ldots & \ldots & \ldots\end{array}$ & 0,967 & 0,973 & 0,997 & 0,989 \\
\hline
\end{tabular}

DESGLOSE DEL SECTOR INDUSTRIAL Y MINERO

\begin{tabular}{|c|c|c|c|c|}
\hline & 1955 & 1957 & 1960 & 1971 \\
\hline 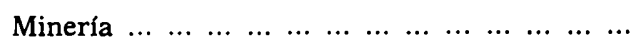 & 1,080 & 1.085 & 1,088 & 1,075 \\
\hline 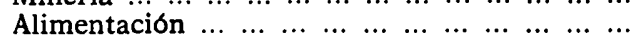 & 0,997 & 1,007 & 1,007 & 1,011 \\
\hline 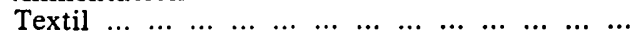 & 1,005 & 0,993 & 0,997 & 0,991 \\
\hline $\begin{array}{lllllllllllllll}\text { Piel } & \ldots & \ldots & \ldots & \ldots & \ldots & \ldots & \ldots & \ldots & \ldots & \ldots & \ldots & \ldots & \ldots & \ldots\end{array}$ & 0,842 & 0,848 & 0,810 & 0,872 \\
\hline 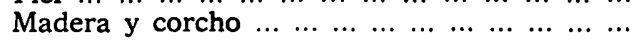 & 0,933 & 0,920 & 0,926 & 0,907 \\
\hline Papel, Prensa y Artes Gráficas $\ldots \ldots \ldots \ldots \ldots$ & 0,936 & 0,944 & 1,010 & 1,004 \\
\hline $\begin{array}{lllllllll}\text { Industrias químicas } & \ldots & \ldots & \ldots & \ldots & \ldots & \ldots & \ldots & \ldots\end{array}$ & 0,937 & 1,027 & 1,019 & 1,017 \\
\hline Siderometalurgia y construcciones metálicas. & 1,018 & 1,024 & 1,031 & 1,018 \\
\hline Cerámica, vidrio y cemento $\ldots \ldots \ldots c c c c$ & 0,957 & 0,922 & 0,951 & 0,954 \\
\hline Edificación $\mathrm{y}$ obras públicas $\ldots \ldots \ldots \ldots \ldots \ldots$ & 1,026 & 1.032 & 1,038 & 0,995 \\
\hline 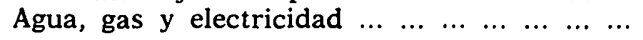 & 0,996 & 1,050 & 1,026 & 1,033 \\
\hline
\end{tabular}

DESGLOSE DE PRODUCCION AGRARIA. 1971

\begin{tabular}{|c|c|c|c|c|c|}
\hline & Alicante & Castellon & Valencia & $\begin{array}{l}\text { Pats Va- } \\
\text { lenciano }\end{array}$ & $\begin{array}{c}\text { Resto de } \\
\text { España }\end{array}$ \\
\hline $\begin{array}{lllllll}\ldots & \ldots & \ldots & \ldots & \ldots & \ldots & \ldots\end{array}$ & 0099 & 0,136 & 0,436 & 0,286 & 1,068 \\
\hline 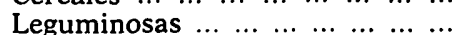 & 0.146 & 0,239 & 0,268 & 0,231 & 1,074 \\
\hline Hortalizas, frutas y patatas ... & 2,716 & 2,712 & 2,361 & 2,526 & 0,854 \\
\hline 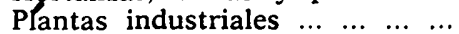 & 0573 & 0,309 & 0,767 & 0,619 & 1,037 \\
\hline $\begin{array}{ccccccccc}\text { Varios } & \ldots & \ldots & \ldots & \ldots & \ldots & \ldots & \ldots & \ldots\end{array}$ & 0,870 & 0,506 & 0,765 & 0,735 & 1,025 \\
\hline 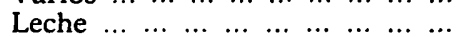 & 0,192 & 0,180 & 0,181 & 0,184 & 1,078 \\
\hline 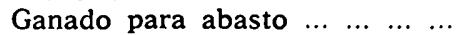 & 0368 & 0,493 & 0,418 & 0,422 & 1,055 \\
\hline Lana, huevos, miel, cera y otros. & 0,552 & 0,761 & 0,821 & 0,740 & 1,025 \\
\hline $\begin{array}{llllllll}\text { Forestal } & \ldots & \ldots & \ldots & \ldots & \ldots & \ldots & \\
\end{array}$ & 0.139 & 0,257 & 0,323 & 0,262 & 1,071 \\
\hline
\end{tabular}


COEFICIENTE DE LOCACION $\propto D \otimes .1971-1960$

\begin{tabular}{|c|c|c|c|c|c|}
\hline & Alicante & Castellon & Valencia & $\begin{array}{l}\text { Pais Va. } \\
\text { lenciano }\end{array}$ & $\begin{array}{c}\text { Resto de } \\
\text { España }\end{array}$ \\
\hline 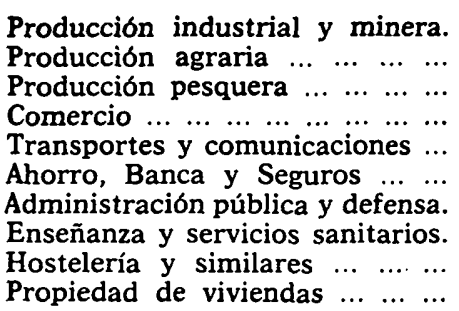 & $\begin{array}{l}1010 \\
0,894 \\
1,501 \\
0840 \\
0,832 \\
1,080 \\
0598 \\
0,717 \\
1,173 \\
1,226\end{array}$ & $\begin{array}{l}1,279 \\
0,837 \\
1,565 \\
1,003 \\
1,454 \\
1,352 \\
1,633 \\
1,297 \\
1,325 \\
1,242\end{array}$ & $\begin{array}{l}1,263 \\
0,703 \\
2,031 \\
1,080 \\
1,051 \\
0,782 \\
1,099 \\
1,242 \\
0,932 \\
1,025\end{array}$ & $\begin{array}{l}1,209 \\
0,752 \\
1,807 \\
1,004 \\
1,019 \\
0,882 \\
0,992 \\
1,023 \\
1,095 \\
1,081\end{array}$ & $\begin{array}{l}0,982 \\
1,039 \\
0,962 \\
0,999 \\
0,998 \\
1,016 \\
1,000 \\
0,992 \\
0,992 \\
0,992\end{array}$ \\
\hline
\end{tabular}

DESGLOSE DEI SECTOR INDUSTRIAL Y MINERO

\begin{tabular}{|c|c|c|c|c|c|}
\hline & Alicante & Castellon & Valencia & $\begin{array}{l}\text { Pats Va- } \\
\text { lenciano }\end{array}$ & $\begin{array}{c}\text { Resto de } \\
\text { España }\end{array}$ \\
\hline 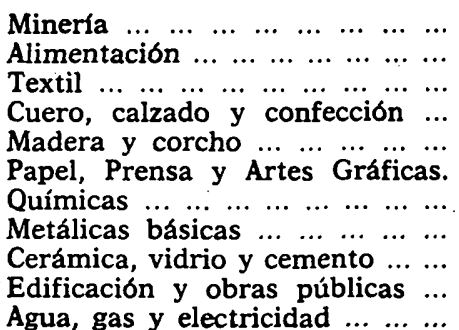 & $\begin{array}{l}1,147 \\
0,598 \\
0924 \\
0,506 \\
0,674 \\
0770 \\
0,785 \\
0,619 \\
0708 \\
1,202 \\
0,831\end{array}$ & $\begin{array}{l}1,150 \\
1,508 \\
1,164 \\
0,349 \\
1,321 \\
0,839 \\
2,480 \\
1,940 \\
1,106 \\
1,924 \\
1,291\end{array}$ & $\begin{array}{l}2,343 \\
1,114 \\
1,028 \\
1,944 \\
1,322 \\
1,284 \\
0,948 \\
1,045 \\
1,136 \\
1,842 \\
0,924\end{array}$ & $\begin{array}{l}1,743 \\
0,958 \\
1,058 \\
0,802 \\
1,115 \\
1,070 \\
1,022 \\
0,929 \\
0,991 \\
1,663 \\
0,905\end{array}$ & $\begin{array}{l}0,988 \\
1,004 \\
0,994 \\
0,770 \\
0,980 \\
0,994 \\
0,998 \\
1,004 \\
1,003 \\
0,959 \\
1,006\end{array}$ \\
\hline
\end{tabular}


REVL-1978, núm. 200. BALLESTER ROS, IGNACIO. NOTAS SOBRE LA EVOLUCION DE LA BASE...

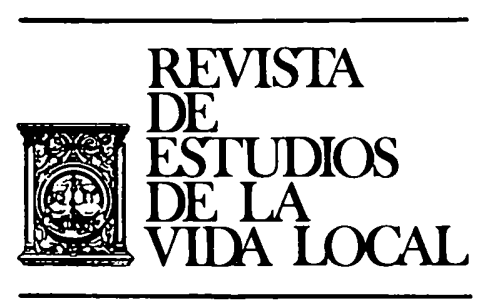

\section{JURISPRUDENCIA}


REVL-1978, núm. 200. BALLESTER ROS, IGNACIO. NOTAS SOBRE LA EVOLUCION DE LA BASE...

REVL-1978, núm. 200. BALLESTER ROS, IGNACIO. NOTAS SOBRE LA EVOLUCION DE LA BASE... 